\title{
A Frequency-Doubling Optoelectronic Oscillator using a Three-Arm Dual-Output Mach-Zehnder Modulator
}

\author{
Yuhua Chong, Chun Yang*, Xianghua Li, and Quanyi Ye \\ School of Electronic Science and Engineering, Southeast University, Nanjing 210096, Jiangsu Province, China
}

(Received October 15, 2013 : accepted November 21, 2013)

\begin{abstract}
This paper proposes a frequency-doubling optoelectronic oscillator employing a novel three-arm dualoutput Mach-Zehnder modulator (MZM). One output of the MZM generates the fundamental-frequency signal which is recycled by the microwave optical fiber link for oscillation, and the other output can generate the frequency-doubled signal. Experiment was conducted using a commercial two-arm MZM, a phase modulator, and an optical fiber link of 89 meters in length. A 19-GHz frequency-doubled signal was successively obtained with fundamental signal suppression more than $36 \mathrm{~dB}$.
\end{abstract}

Keywords : Optoelectronic oscillator, Frequency doubling, Mach-Zehnder modulator, Microwave generation OCIS codes : (060.4510) Optical communications; (230.4110) Modulators; (250.0250) Optoelectronics

\section{INTRODUCTION}

Optoelectronic oscillators (OEOs) are promising highpurity microwave sources for various applications such as clock recovery $[1,2]$, format transformation [3, 4], and radar systems $[5,6,7]$. Usually, the oscillation frequency of a conventional OEO is mainly restricted by the bandwidth of components in the optoelectronic feedback loop, especially the electro-optic modulator and electrical components. In order to obtain a higher oscillating frequency, a few studies focusing on frequency-doubling OEOs have been reported [8-11]. Sakamoto et al. firstly proposed a frequencydoubling OEO using a pull-push Mach-Zehnder modulator (MZM) biased at the null transmission point (NTP) [8]. However, an amplifier and a high-selective bandpass filter have to work at the doubled frequency. Furthermore, a frequency divider with large insert loss has to be used. In [9], M. Shin et al. proposed a frequency-doubling OEO based on the wavelength dependence of the halfwave voltage of the MZM. In this case, two lasers with different wavelengths $(1310$ and $1550 \mathrm{~nm})$ are required to configure the system. Recently, frequency-doubling OEOs based on a dual-parallel Mach-Zehnder modulator (DPMZM) in conjunction with optical sideband modification devices were introduced $[10,11]$. One method used a chirped fiber Bragg grating to modify the relative phase between sidebands.
But a highly stable tunable laser is required due to the high-sensitive wavelength dependence of phase characteristic of the grating [10]. Another method utilized stimulated Brillouin scattering (SBS) to realize the high-selective electrical filter to suppress one sideband [11]. However, it is not easy to configure a low phase noise OEO, because Stokes noise caused by SBS effect can deteriorate phase noise.

In this paper, we propose a frequency-doubling OEO based on a three-arm dual-output MZM. One output generates the fundamental frequency for sustaining the oscillation of the OEO, and the other output is designed to generate the frequency-doubled signal. The proposed configuration does not need optical sideband modification devices and potentially can be monolithically integrated. In the following sections, the principle and configuration of the proposed frequency-doubling OEO are introduced first. And then experimental results are presented and discussed.

\section{STRUCTURE AND PRINCIPLE}

The structure of the three-arm MZM is composed of a phase-modulating arm-a, a non-modulating arm-b, and a bias arm-c constructed in parallel. The arm-a and arm-b connected with two $\mathrm{Y}$ branches operates as a two-arm $\mathrm{MZM}_{1}$. Output of the $\mathrm{MZM}_{1}$ is split where the upper output

*Corresponding author: yangchun_seu@163.com 
(port-e) is connected to $\mathrm{PD}_{2}$ and the lower output (arm-d) is combined with arm-c via another $\mathrm{Y}$ branch. If the $\mathrm{MZM}_{1}$ is biased at the maximum transmission point (MaxTP) where arm-a and arm- $b$ are in phase, the doubled frequency of the modulation electrical signal on $\mathrm{MZM}_{1}$ is obtained at the output of the $\mathrm{PD}_{2}$. The port-f is the output for the oscillation signal of fundamental-frequency which is recycled to the electrical port of the three-arm MZM through a long optical fiber link, followed by an electrical amplifier and a fundamental-frequency selective filter. The operation principle can be studied firstly by considering a modulation signal $V_{m}=V_{a} \sin \left(\omega_{m} t\right)$ driving the $\mathrm{MZM}_{1}$ which is biased at MaxTP. Assuming the electric field of the light source is $E_{\text {in }}=E_{0} \exp \left(j \omega_{0} t\right)$. The electric field at port-e can be expressed as

$$
E_{e}(t)=\frac{1}{4} E_{\text {in }} \exp \left[j \beta \sin \left(\omega_{m} t\right)+j \phi_{1}\right]+\frac{1}{4} E_{\text {in }}
$$

where $\omega_{0}=2 \pi f_{0}$ and $\omega_{m}=2 \pi f_{m}$ are the angular frequency of the light source and the modulation microwave signal respectively, $\beta=\pi V_{a} / V_{\pi}$ is the modulation index of the $\mathrm{MZM}_{1}, V_{\pi}$ is the halfwave voltage of the $\mathrm{MZM}_{1}, f_{1}$ is the bias-angle of the $\mathrm{MZM}_{1}$ which is zero under MaxTP.

The photo-current generated by $\mathrm{PD}_{2}$ can be expressed as

$$
\begin{aligned}
& I_{2}(t)=\rho_{2}\left|E_{e}(t)\right|^{2} / 2 \\
& =\frac{1}{8} \rho_{2} P_{0}\left[1+J_{0}(\beta)+2 \sum_{m=1}^{\infty} J_{2 m}(\beta) \cos \left(2 m \omega_{m} t\right)\right]
\end{aligned}
$$

where $r_{2}$ is the responsivity of $\mathrm{PD}_{2}, P_{0}=\left|E_{0}\right|^{2} / 2$ is the power of the light source, $J_{\mathrm{m}}(\cdot)$ is the $m$-th order Bessel function of the first kind. It can be seen from Eq. (2) that the output current contains a DC component and even-order harmonic components, while the fundamentalfrequency signal is eliminated. A frequency-doubled signal is obtained while the fourth and higher order harmonics are suppressed because they exceed the bandwidth of the $\mathrm{PD}_{2}$. In order to generate a fundamental-frequency signal at $\mathrm{PD}_{1}$ to sustain the oscillation of the OEO, the arm-d is beat with an un-modulated optical carrier contained in arm-c which is biased quadrature to arm-a. The electric field at port-f can be expressed as

$$
E_{f}(t)=\frac{\sqrt{2}}{8} E_{i n} \exp \left[j \beta \sin \left(\omega_{m} t\right)\right]+\frac{\sqrt{2}}{8} E_{i n}+\frac{1}{2} E_{i n} \exp \left(j \phi_{2}\right)
$$

where $f_{2}$ is the phase difference of the optical carrier between arm-a and arm-c. The photo-current generated by $\mathrm{PD}_{1}$ can be expressed as

$$
\begin{aligned}
& I_{1}(t)=\frac{\rho_{1} P_{0}}{16}\left\{5+J_{0}(\beta)+2 \sqrt{2}\left[1+J_{0}(\beta)\right] \cos \left(\phi_{2}\right)\right. \\
& +4 \sqrt{2} \sin \left(\phi_{2}\right) \sum_{m=1}^{\infty} J_{2 m-1}(\beta) \sin \left[(2 m-1) \omega_{m} t\right] \\
& \left.\left[2+4 \sqrt{2} \cos \left(\phi_{2}\right)\right] \sum_{m=1}^{\infty} J_{2 m}(\beta) \cos \left(2 m \omega_{m} t\right)\right\}
\end{aligned}
$$

where $r_{1}$ is the responsivity of $\mathrm{PD}_{1} . I_{1}(t)$ contains a DC component, odd-order harmonic components, and evenorder harmonic components. After transmission through the bandpass filter (BPF), the output only contains the fundamental frequency $\omega_{m}$ with higher-order harmonic components filtered out. The amplitude of the fundamental-frequency signal mainly depends on $\phi_{2}$. In order to obtain the highest signal gain, $f_{2}$ is adjusted to $(\mathrm{n}+1 / 2) \mathrm{p}$ which are called quadrature points. The DC term $\sqrt{2} / 8\left[1+J_{0}(\beta)\right] \cos \left(\phi_{2}\right)$ indicates a cosine transfer curve. Therefore the bias between arm-a and arm-c can be tracked at the half-power point on the transfer curve. When the open-loop gain is larger than unity, OEO will generate a fundamental frequency and a frequency-doubled microwave signal simultaneously.

\section{EXPERIMENTAL AND DISCUSSION}

Experiment based on the structure shown in Fig. 1 was conducted. The three-arm MZM was constructed by a single-arm-drive Z-cut MZM (Thorlabs LN58) serving as $\mathrm{MZM}_{1}$ and a phase modulator (Thorlabs LN53) as arm-c which were connected by three $3-\mathrm{dB}$ polarization maintaining fiber couplers. The three-arm MZM was placed in a wooden box in order to isolate it from environmental interference. Both $\mathrm{PD}_{1}$ and $\mathrm{PD}_{2}$ (Optilab PD-30) have a -3 $\mathrm{dB}$ bandwidth of $28 \mathrm{GHz}$ and a responsivity of $0.8 \mathrm{~A} / \mathrm{W}$. The microwave amplifier has a maximum gain of $50 \mathrm{~dB}$. The bandpass filter with $9.5-\mathrm{GHz}$ center frequency has a $-3 \mathrm{~dB}$ bandwidth of $20 \mathrm{MHz}$. The length of the optical fiber is about $89 \mathrm{~m}$ corresponding to FSR of $2.3 \mathrm{MHz}$ which can be seen in Fig. 3 .

In the experiment, the $\mathrm{MZM}_{1}$ was carefully biased to MaxTP by manually adjusting the voltage at bias- 1 to minimize the fundamental signal at the output of $\mathrm{PD}_{2}$. Thanks to the high stability of the commercial $\mathrm{MZM}_{1}$ used and the wooden box, the bias-1 can be held for a few hours. Nevertheless, a low-frequency pilot tone based feedback control should be applied to hold the MATP at bias-1 in real time [12]. The arm-c was held on quadrature with respect to the arm-a by a bias controller based on monitoring the average optical intensity at port-f. A fundamental microwave signal at $9.5 \mathrm{GHz}$ and a frequency-doubled signal at 19

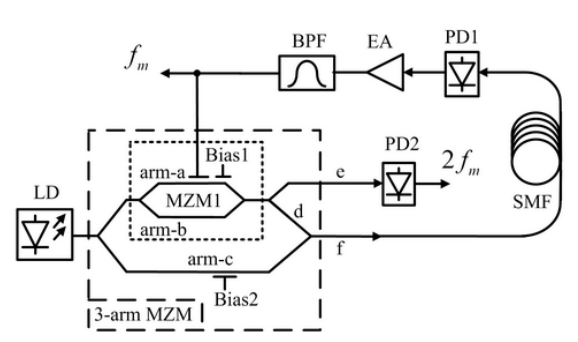

FIG. 1. The structure of the proposed frequency-doubling OEO. LD: laser diode; PD: photodetector; SMF: single-mode fiber; EA: electrical amplifier; BPF, bandpass filter. 


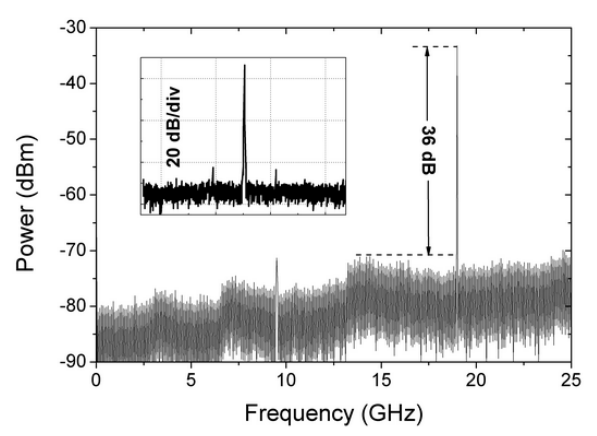

FIG. 2. Electrical spectrum of the frequency-doubled signal at 19 GHz. Inset: enlarged spectrum at resolution bandwidth of 33 $\mathrm{kHz}$ and $15 \mathrm{MHz}$ Span.

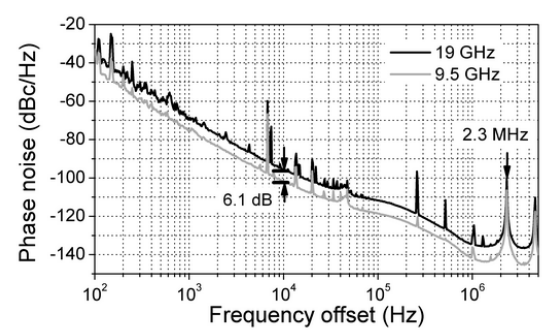

FIG. 3. Phase noise spectra of the generated 9.5 and $19 \mathrm{GHz}$ signal.

$\mathrm{GHz}$ were generated at $\mathrm{PD}_{1}$ and $\mathrm{PD}_{2}$, respectively.

Figure 2 shows the RF spectrum of the frequencydoubled signal measured by an electrical spectrum analyzer (Agilent E4440A). It clearly shows a pure frequency-doubled signal at $19 \mathrm{GHz}$ is obtained which is $36-\mathrm{dB}$ greater than the fundamental-frequency component.

The phase noises of the fundamental and frequencydoubled signals shown in Fig. 3 were measured by using a signal source analyzer (Agilent 5052A) which covers a frequency range below $7 \mathrm{GHz}$. To measure the phase noise of the generated 9.5- and $19-\mathrm{GHz}$ signals, the microwave signals were down-converted to an intermediate frequency by mixing with a reference signal which is generated by a signal generator (Agilent E8267D). As can be seen, the phase noises of 9.5- and 19-GHz signals are $-96 \mathrm{dBc} / \mathrm{Hz}$ and $-102.1 \mathrm{dBc} / \mathrm{Hz}$ respectively at $10 \mathrm{kHz}$ frequency offset. The phase noises of the generated signals are $10-\mathrm{dB}$ greater than those of the reference signals, ensuring reliable measurements of phase noise. The phase noise of the $19 \mathrm{GHz}$ signal is approximately 6-dB greater than that of the $9.5 \mathrm{GHz}$ signal, which agrees well with the theoretical prediction of $20 \log _{10} 2=6 \mathrm{~dB}$. Sidemodes of the OEO with constant frequency spacing of $2.3 \mathrm{MHz}$ are observed and are lower than $-100 \mathrm{dBc} / \mathrm{Hz}$.

\section{CONCLUSION}

A frequency-doubling OEO has been proposed employing a three-arm MZM configured by utilizing a commercial single-arm-drive MZM and a phase modulator. Compared with previously reported frequency-doubling OEOs, the proposed system has two advantages: 1) there is no need to employ optical sideband modification devices in the loop; 2) the three-arm MZM which is the key element of the proposed system can be monolithically integrated to obtain high stability. The frequency-doubled signal at 19 $\mathrm{GHz}$ was obtained experimentally and was 36-dB greater than the fundamental-frequency signal.

\section{REFERENCES}

1. H. Tsuchida, "Simultaneous prescaled clock recovery and serial-to-parallel conversion of data signals using a polarization modulator-based optoelectronic oscillator," J. Lightwave Technol. 27, 3777-3782 (2009).

2. S. L. Pan and J. P. Yao, "Optical clock recovery using a polarization-modulator-based frequency-doubling optoelectronic oscillator," J. Lightwave Technol. 27, 3531-3539 (2009).

3. J. P. Yao, "Microwave photonics," J. Lightwave Technol. 27, 314-225 (2009).

4. L. Huo, Y. Dong, C. Y. Lou, and Y. Z. Gao, "Clock extraction using an optoelectronic oscillator from highspeed NRZ signal and NRZ-to-RZ format transformation," IEEE Photon. Technol. Lett. 15, 981-983 (2003).

5. X. S. Yao and L. Maleki, "Converting light into spectrally pure microwave oscillation," Opt. Lett. 21, $483-485$ (1996).

6. A. J. Seeds, "Microwave photonics," IEEE Trans. Microwave Theory Tech. 50, 877-887 (2002).

7. J. Hong and C. Yang, "Eeffects of the biasing voltage of modulator on the phase noise of opto-electronic oscillator," Microwave and Opt. Technol. Lett. 54, 689-692 (2012).

8. T. Sakamoto, T. Kawanishi, and M. Izutsu, "Optoelectronic oscillator using push-pull Mach-Zehnder modulator biased at null point for optical two-tone signal generation," in Proc. Conf. Lasers Electro-Optics (Baltimore, Maryland, May 2005), pp. 877-879.

9. M. Shin, V. S. Grigoryan, and P. Kumar, "Frequencydoubling optoelectronic oscillator for generating highfrequency microwave signals with low phase noise," Electron. Lett. 43, 242-244 (2007).

10. W. Lixian, Z. Ninghua, L. Wei, and L. Jianguo, "A frequency-doubling optoelectronic oscillator based on a dual-parallel Mach-Zehnder modulator and a chirped fiber Bragg grating," IEEE Photon. Technol. Lett. 23, 1688-1690 (2011).

11. B. Yang, X. Jin, H. Chi, X. Zhang, S. Zheng, S. Zou, H. Chen, E. Tangdiongga, and T. Koonen, "Optically tunable frequency-doubling Brillouin optoelectronic oscillator with carrier phase-shifted double sideband modulation," IEEE Photon. Technol. Lett. 24, 1051-1053 (2012).

12. E. I. Ackerman and C. H. Cox III, "Effect of pilot tonebased modulator bias control on external modulation link performance," in Proc. IEEE International Topical Meeting on Microwave Photonics (Oxford, Sep. 2000), pp. 121-124. 